INCAST 2008- 105

\title{
PREDICTION OF TOTAL PRESSURE CHARACTERISTICS IN THE SETTLING CHAMBER OF A SUPERSONIC BLOWDOWN WIND TUNNEL
}

\author{
S R Bhoi ${ }^{1}$ and G K Suryanarayana ${ }^{2}$ \\ 1 National Trisonic Aerodynamic Facilities, National Aerospace Laboratories, Bangalore, India, \\ satyajeet@css.nal.res.in \\ 2 National Trisonic Aerodynamic Facilities, National Aerospace Laboratories, Bangalore, India
}

\begin{abstract}
In the design of control system of a wind tunnel, it is important to understand the importance of operations of mechanical systems in order to ensure that any emergencies that may arise during the running of the wind tunnel are properly handled by the system. In the NAL $0.6 \mathrm{~m}$ blowdown wind tunnel, it is proposed to incorporate a Variable Mach number Flexible Nozzle (VMFN), using which the tunnel can be started at a low Mach number and the required test Mach number (up to 4) can be reached by continuously (on-line) changing the nozzle contour using an Electroservohydraulic drive. Before stopping the tunnel, the VMFN would be reverted to the initial low Mach number condition. During the entire operation of the tunnel in such a scenario, it is essential that the Pressure Regulating Valve (PRV) maintains the specified or minimum stagnation pressure in the test section by following a safe trajectory. In order to determine a safe trajectory, a basic understanding of the variation of stagnation pressure in the settling chamber is called for, before the PRV control system is designed. In this paper, the problem is formulated based on quasi-steady isentropic equations and programmed in C language to predict the timehistories of settling chamber pressure and storage tank pressure for a given trajectory of the opening of PRV, as the VMFN throat is changed from Mach 1 to Mach 4 condition and vice versa. In order to validate the present methodology, the PRV trajectory measured during an experiment in the NAL 0.6m wind tunnel was used as input to the program and the measured time-histories of settling chamber pressure and storage tank pressure are compared with the predicted values. Predictions of settling chamber pressure and the storage tank pressure relevant to VMFN operation indicate that settling chamber pressure rapidly builds up towards the storage tank pressure, apparently due to the constriction offered at the VMFN throat as VMFN throat is changed from $M=1$ contour to $M=4$ contour during the start of the run. Minimum PRV opening area has been predicted to maintain the settling chamber pressure higher than the estimated minimum value at high Mach numbers. For smaller openings of the PRV (< $\left.0.065 \mathrm{~m}^{2}\right)$, the predicted pressure falls below the minimum and is hence unsafe. From the predicted time history of free-stream dynamic pressure $(q)$, it is noted that alarming increase in $q$ can occur during starting and stopping transients, which can damage a test model in the tunnel, balance and such other components. Therefore, the design of test object and support system need to be adequately designed.
\end{abstract}




\begin{tabular}{|ll|}
\hline \multicolumn{2}{|l|}{ Nomenclature } \\
$a$ & speed of sound \\
$A$ & area \\
& \\
$M$ & mass flow \\
$M$ & Mach number \\
$n$ & expansion index \\
$p$ & pressure \\
$P$ & total pressure \\
$q$ & dynamic pressure \\
$r$ & pressure ratio \\
$R$ & gas constant \\
$t$ & time \\
$T$ & temperature \\
$u$ & velocity \\
$V$ & volume \\
$\gamma$ & ratio of specific heats \\
$\rho$ & density \\
& \\
Subscripts \\
$S$ & storage tank \\
$S C$ & settling chamber \\
$t$ & throat \\
$v$ & valve \\
0 & initial conditions \\
$a t m$ & Atmospheric \\
$m i n$ & minimum \\
max & maximum \\
Superscript \\
$*$ & Sonic condition \\
& \\
\hline
\end{tabular}

\section{INTRODUCTION}

As a part of NTAF augmentation programme, it is proposed to augment the testing capability of the NAL $0.6 \mathrm{~m}$ blowdown wind tunnel from transonic Mach numbers to supersonic Mach numbers up to 4.0. For this purpose, a Variable Mach number Flexible Nozzle (VMFN), driven by a single hydraulic jack is being developed. In blow-down wind tunnels, very high starting and stopping loads occur while testing at high supersonic Mach numbers (Ref. 1). In the $0.6 \mathrm{~m}$ wind tunnel, it is proposed to start the VMFN at a low Mach number (say, $M=1.0$ ) and a low stagnation pressure and reach the test Mach number (up to 4.0) to avoid starting loads. After completion of tests at the desired Mach number, the VMFN would be brought back to Mach 1.0 condition before shutting down the run, in order to avoid stopping loads. During the entire operation, the second throat would be kept fixed at a position corresponding to the starting condition at Mach 1.2 so that the normal shock during starting of the tunnel is anchored beyond the second throat at all supersonic Mach numbers.

During the VMFN operation as described above, two important issues arise. Firstly, after the flow stabilizes at Mach 1.0 during start, it is essential to maintain a minimum stagnation pressure in the settling chamber using the Pressure Regulating Valve (PRV), to avoid supersonic flow breakdown in the test section. Secondly, since the storage tank pressure drops continuously as blowdown progresses, the PRV must be close-loop controlled to maintain constant dynamic pressure in the free-stream during the useful run. The available run time in the wind tunnel is governed by the mass flow rate, which in turn depends on the VMFN throat as well as opening area at the PRV, in addition to other parameters such as storage tank pressure, storage tank volume, losses at the PRV, etc. Thus, the problem of operation of VMFN involves the dynamics of Mach number change and that of the PRV trajectory, besides the other fixed parameters related to the test facility. Further, in order to minimize the wastage of compressed air from the storage tank, it is required to reach the test Mach number in the quickest possible time, after the flow is started at Mach 1.0. However, during the entire blowdown, the dynamic pressure in the test section should not be undesirably high, since this affects the design of the model and the balance in supersonic Mach number tests. It is therefore necessary to understand the stagnation pressure characteristics in the settling chamber, as a function of the VMFN throat (equivalent to Mach number) as well as the PRV trajectory.

In this paper, the problem is formulated based on quasi-steady isentropic equations to study the stagnation pressure characteristics in the settling chamber and the pressure drop in the storage tank for various opening and closing trajectories of the PRV when the Mach number is varied from 1.0 to 4.0 and vice versa. A computer program in $\mathrm{C}$ language is presented to predict the settling chamber pressure characteristics. The program is validated with results from tests conducted in the $0.6 \mathrm{~m}$ wind tunnel at few subsonic Mach numbers. Predictions are made for the settling chamber pressure characteristics relevant to VMFN operation.

\section{OBJECTIVES AND PARAMETERS OF THE PROBLEM}

The desired output from the present exercise is to predict the following with respect to VMFN operation.

1. Effect of change of Mach number from 1.0 to 4.0 on the settling chamber pressure for a fixed position of PRV 
Effect of PRV trajectory on the settling chamber pressure and test section dynamic pressure as a function of Mach number.

3. Maximum available run-time at Mach 4.0 using various numbers of storage tanks (minimum volume of $708 \mathrm{~m}^{3}$ to maximum of $3600 \mathrm{~m}^{3}$ )

4. To study whether an optimum trajectory for the PRV can be predicted, which ensures that

a) after the flow stabilizes at Mach 1.0 condition, the stagnation pressure is always higher than the minimum safe limit to avoid flow breakdown at any supersonic Mach number,

b) wastage of compressed air is minimized by maximizing the useful run time in a run,

c) the dynamic pressure in the test section is not undesirably high during transition from $M=1.0$ to 4.0 and vice versa and is constant during the useful run time.

The parameters that decide the above issues are the volume of storage tanks (maximum $3600 \mathrm{~m}^{3}$ ), the maximum initial storage tank pressure $(11.24$ bar), temperature $(300 \mathrm{~K})$, volume of the settling chamber (up to VMFN throat for supersonic test conditions and up to second throat for subsonic and transonic test conditions), first throat area (at the VMFN), flow area and losses at the PRV.

\section{FORMULATION OF THE PROBLEM}

Fig 1 shows a schematic of the $0.6 \mathrm{~m}$ wind tunnel, incorporating the VMFN. The governing equations for the mass flow rate through the PRV depend on whether the valve is choked or not (Ref 2), which in turn depends on the upstream and downstream conditions across the PRV and the opening area at the PRV. If the flow is choked, the mass flow is independent of pressure in the settling chamber and the variation of settling chamber pressure and the storage tank pressure are related through coupled linear equations. If the PRV is not choked, the entry mass flow depends on settling chamber pressure as well as the storage tank pressure, and the governing equations become coupled non-linear differential equations. These equations maybe solved using standard numerical methods. The following assumptions have been made

1. Polytropic expansion of air in the storage tank.

2. Stagnation temperature in the storage tank and the settling chamber are identical.

3. When the PRV is not choked, static pressure at the valve equals 0.96 times the settling chamber pressure.

4. When the VMFN throat is not choked, $5 \%$ loss of total pressure occurs at the throat.

5. Quasi-steady one-dimensional flow.

6. Isentropic flow equations are valid.

Based on these assumptions and the continuity equations across the PRV and the throat the differential equations for the $P_{S C}$ and $P_{S}$ variation have been derived (Ref 3 ).

The differential equations are summarized as

$$
\begin{gathered}
\frac{d P_{s}}{d t}=-\frac{n F\left(r_{v}\right)}{V_{s}} \sqrt{\gamma R T_{s 0}} \frac{1}{P_{s o}^{\frac{n-1}{n}}} P_{s}^{\frac{2 n-1}{n}} A_{v} \\
\frac{1}{\sqrt{\gamma R T_{s o}}} \frac{d P_{s c}}{d t}+\frac{A_{t}}{V_{s c}} P_{s c} F\left(r_{t}\right)=\frac{P_{s}}{V_{s c}} A_{v}(t) F\left(r_{v}\right)
\end{gathered}
$$

where

$$
\begin{aligned}
& F\left(r_{v}\right)=C_{1} \quad \text { if valve is choked } \\
& =f\left(r_{v}\right) \quad \text { if valve is unchoked } \\
& \mathrm{F}\left(\mathrm{r}_{\mathrm{t}}\right)=C_{1} \quad \text { if throat is choked } \\
& =f\left(r_{t}\right) \quad \text { if throat is unchoked } \\
& C_{1}=\left(\frac{2}{\gamma+1}\right)^{\frac{\gamma+1}{2(\gamma-1)}}
\end{aligned}
$$




$$
\begin{aligned}
& f(r)=\left[\frac{2}{\gamma-1} r^{\frac{2}{\gamma}}\left(1-r^{\frac{\gamma-1}{\gamma}}\right)\right]^{\frac{1}{2}} \\
& r_{v}=0.96 P_{s c} / P_{s} \\
& r_{t}=p_{a t m} /\left(0.95 P_{s c}\right)
\end{aligned}
$$

(1) and (2) constitute a pair of coupled non-linear differential equations for the variation of $P_{s}$ and $P_{s c}$ with time respectively

\section{RESULTS AND DISCUSSIONS}

\subsection{Validation of the method}

Subsonic Mach numbers in the test section of the $0.6 \mathrm{~m}$ tunnel are obtained by choking the second throat and by setting $P_{s c}$. The existing tunnel does not have provision to vary $A_{t}$. So, it is possible to validate the present method only at subsonic/transonic $M$. Figs. 2 and 3 show the comparison of the predicted $P_{S C}$ and $P_{S}$ characteristics with the test run data for $M=0.5$. Good comparison between the predicted and experimental results can be seen, validating the present methodology.

\subsection{Minimum stagnation pressures in $0.6 \mathrm{~m}$ wind tunnel at supersonic mach numbers}

\section{Table 1: Estimated minimum $P_{S C}$ for various Mach numbers}

\begin{tabular}{rrrrrrrr}
\hline$M$ & 1 & 1.2 & 2 & 2.5 & 3 & 3.8 & 4 \\
\hline$P_{\min }$ (bar) & 1.72 & 1.72 & 2.07 & 2.41 & 4.14 & 6.21 & 7.58 \\
\hline
\end{tabular}

In order to achieve supersonic $M$ in the test section, the second throat must be set to the 'starting' condition and the appropriate $P_{S C}$ must be chosen to enable the normal shock at start to pass through the test section and locate itself in a stable position downstream of the second throat. After the flow starts, the second throat may be reduced to the 'running' condition, which enables supersonic operation at a lower $P_{S C}$ and hence increases the run time. If $P_{S C}$ falls below this minimum value $P_{\min }$, the test section flow breaks down into a subsonic condition, resulting in large oscillatory loads. In case of VMFN, since the flow is started at $M=1.0$ with sufficiently wide second throat, $P_{\min }$ would be corresponding to the 'running' pressure. However, since $P_{\min }$ is not a priori known, typical starting pressure in the $1.2 \mathrm{~m}$ wind tunnel is used as reference. Table 1 shows the values of $P_{\min }$ corresponding to $M$ estimated for the $0.6 \mathrm{~m}$ tunnel. It is desirable to have the $P_{S C}$ marginally higher than the $P_{\min }$, at any time during the blowdown at high $M$. The $P_{\min }$ values at intermediate $M$ are interpolated from the above values.

\subsection{Pressure build-up characteristics}

Table 2: Predicted $q_{\max }$ for various PRV openings

\begin{tabular}{rrrr}
\hline$A_{v}\left(\mathrm{~m}^{2}\right)$ & $t(\mathrm{~s})$ & $q_{\max }(\mathrm{bar})$ & $M_{q \max }$ \\
\hline 0.065 & 5 & 0.876 & 2.06 \\
0.079 & 4.5 & 1.062 & 2.06 \\
0.093 & 4.13 & 1.247 & 2.06 \\
0.139 & 3.27 & 1.884 & 2.39 \\
0.186 & 2.7 & 2.573 & 2.01 \\
0.232 & 2.23 & 3.130 & 1.73 \\
0.279 & 1.8 & 3.500 & 1.62 \\
0.325 & 1.0 & 3.757 & 1.56 \\
0.390 & 0.71 & 4.000 & 1.50 \\
\hline
\end{tabular}


Fig 4 shows the PRV trajectory for a maximum $A_{v}$ of $0.046 \mathrm{~m}^{3}$ and profile of $M$ used as inputs to the program to compute the variation of the $P_{S C}$ and $P_{S}$ with $V_{S}$ corresponding to all tanks and $P_{S O}=11.24 \mathrm{bar}$. Fig. 5 shows the $P_{S}$ and $P_{S C}$ characteristics obtained by simulation. It is observed that $P_{S C}$ rapidly builds up towards $P_{S}$ and reaches a maximum value of 9.65 bar and remains nearly constant up to $16 \mathrm{~s}$. By predicting $P_{S C}$ for various $A_{v}$ values from $A_{v \min }=0.028 \mathrm{~m}^{2}$ to $A_{v \max }=0.390 \mathrm{~m}^{2}$ it is observed that larger $A_{v}$ results in a faster build up of $P_{S C}$, as expected. Minimum $A_{v}=0.065 \mathrm{~m}^{2}$ is required to maintain $P_{S C}$ above $P_{\min }$ values at high $M$ during the starting transient. Fig 6 shows the $q$ characteristics for various $A_{v}$. For any $A_{v}$, a peak in $q\left(q_{\max }\right)$ is observed during transition from $M=1.0$ to $M=4.0$. Also, $q_{\max }$ increases with increase in $A_{v}$. The table 2 summarizes the time taken for the $P_{S C}$ to reach 8.27 bar for various $A_{v}$, the magnitude of $q_{\max }$ and $M$ at $q_{\max }$. From $A_{v}=0.027 \mathrm{~m}^{2}$ to $A_{v}=0.065 \mathrm{~m}^{2}, M$ at $q_{\max }$ increases from about 1.5 to 2.06. For $A v=0.093 \mathrm{~m}^{2}$ to $0.39 \mathrm{~m}^{2}, M$ at $q_{\max }$ decreases from 2.06 to about 1.5 . It is noted from the table that if the PRV opening is not controlled, or if the PRV gets into a 'runaway' condition, the instantaneous $q_{\max }$ values can be very large, resulting in damage to model, balance etc.

\subsection{Run time pressure characteristics}

Table 3: Predicted PRV trajectory and useful runtime for various storage tank volumes

\begin{tabular}{rrrrrrr}
\hline No.of Storage tanks & $\begin{array}{r}A_{v 1} \\
\left(\mathrm{~m}^{2}\right)\end{array}$ & $\begin{array}{r}t_{l} \\
(\mathrm{~s})\end{array}$ & $\begin{array}{r}A_{v 2} \\
\left(\mathrm{~m}^{2}\right)\end{array}$ & $\begin{array}{r}d t \\
(\mathrm{~s})\end{array}$ & $\begin{array}{r}d A_{v} / d t \\
\left(\mathrm{~m}^{2} / \mathrm{s}\right)\end{array}$ & $\begin{array}{r}\text { Maximum runtime } \\
(\mathrm{s})\end{array}$ \\
\hline 1 & 0.065 & 4.9 & 0.038 & 0.2 & 0.000891 & 11 \\
2 & 0.065 & 4.9 & 0.031 & 0.2 & 0.000408 & 30 \\
3 & 0.065 & 4.9 & 0.29 & 0.2 & 0.000222 & 55 \\
4 & 0.065 & 4.9 & 0.29 & 0.2 & 0.000148 & 80 \\
5 & 0.065 & 4.9 & 0.29 & 0.2 & 0.000111 & 100 \\
\hline
\end{tabular}

The run time $P_{S C}$ and the maximum run time available are influenced by the $V_{S}$ and the drop in $P_{S}$. In the test facility, the air is stored in a series of five interconnected storage tanks of $708 \mathrm{~m}^{3}$ capacity each and not all tanks may be available to the $0.6 \mathrm{~m}$ tunnel. The effect of increased $V_{S}$ from minimum ( 1 tank) to maximum ( 5 tanks) is studied. $\mathrm{P}_{\mathrm{S} 0}$ has been taken as $11.24 \mathrm{bar}$ though the tests can be done at lower pressure also. Run time $P_{S C}$ characteristics have been evaluated considering $M=1$ at start with rate of change of $0.75 \mathrm{M} / \mathrm{s}$. For the present studies it is assumed that the maximum run time would be up to the time till $P_{S} \geq 1.10 P_{S C}$. The control of PRV switches to the pressure mode from position mode when the pressure builds up to $P_{S C}$. For example if the $P_{S C}$ builds up more than 8.27 bar at $M=4.0$, the pressure mode will reduce the PRV area to maintain $P_{S C}$ at 8.27 bar. A typical PRV trajectory is shown in Fig 7 . It is to be noted that the PRV opening gradually increases to compensate for the continuous drop in the $P_{S}$ as the run advances. The parameters defined in Fig. 7 for regulated PRV have been obtained by trial and error and are tabulated in table 3 for various numbers of storage tanks to buildup and maintain the constant $P_{S C}$ of 8.27 bar at $M=4.0$.

\subsection{Pressure drop characteristics}

Table 4: Predicted $q_{\text {max }}$ during stopping of the run

\begin{tabular}{rrrr}
\hline$d A_{\downarrow} / d t\left(\mathrm{~m}^{2} / \mathrm{s}\right)$ & $t(\mathrm{~s})$ & $q_{\max }($ bar $)$ & $M_{\text {qmax }}$ \\
\hline 0.008 & 3 & 1.132 & 2.78 \\
0.011 & 3 & 1.091 & 2.82 \\
0.016 & 3 & 1.022 & 2.93 \\
\hline
\end{tabular}

During the stopping transient, the PRV is commanded to close simultaneously as the nozzle contour is changed from Mach 4.0 to 1.0 at the rate of $0.75 \mathrm{M} / \mathrm{s}$ after completion of the test. The $P_{S C}$ characteristics for different rates of closing the PRV indicate that the $P_{S C}$ is always above the $P_{\min }$ irrespective of PRV closing rate. The $P_{S C}$ drops to the $P_{a t m}$ in about $3 \mathrm{~s} . q$ peaks to a high value, $q_{\max }$ during transition from Mach 4.0 to 1.0 during stopping also. The $q_{\max }$ is higher than that during starting of the run. Higher closing rate causes a marginal reduction in $q_{\max }$ but at a marginally higher $M$. The time for the $P_{S C}$ to drop to ambient, $q_{\max }$ and the $M$ at $q_{\max }$ are tabulated in table 4 for various rates of closing the PRV. 


\section{CONCLUDING REMARKS}

Predictions of the $P_{S C}$ characteristics relevant to VMFN operation in $0.6 \mathrm{~m}$ wind tunnel are presented. The predictions are validated with experimental data at subsonic $M$. The simulation has been used to predict the PRV opening trajectory necessary to maintain $P_{S C}$ above $P_{\min }$ value required to maintain the supersonic flow in the test section at any $M$. During transition from $M=1.0$ to $M=4.0$ and vice versa, peak values of $q$ of as much as $2.068 \mathrm{bar}$ are indicated. Thus it appears that even though the use of VMFN may alleviate start stop loads, the need to design wind tunnel models for loads arising from high $q$ still continues

\section{ACKNOWLEDGEMENTS}

Useful discussions with Dr S N Seshadri, former Head, NTAF, NAL and Shri G Rajendra, Emeritus Scientist, NTAF, NAL are acknowledged with thanks. The efforts of staff of instrumentation and controls group of NTAF in acquiring data in the $0.6 \mathrm{~m}$ tunnel for the generation of experimental data for validation purposes are gratefully acknowledged.

\section{REFERENCES}

[1] Pope, A. and Goin, K., High-Speed Wind Tunnel Testing, Krieger Publishing Company, Wiley, New York, 1965

[2] Anderson, John D., Jr, Modern Compressible Flow: with Historical Perspective, McGraw-Hill Book Co., New York, 1982.

[3] Satyajeet Ratan Bhoi and Suryanarayana, G K., Prediction of Pressure Characteristics in Settling Chamber of 0.6m Wind Tunnel for Supersonic Testing, PDNT 0809, National Aerospace Laboratories, Bangalore, March 2008 


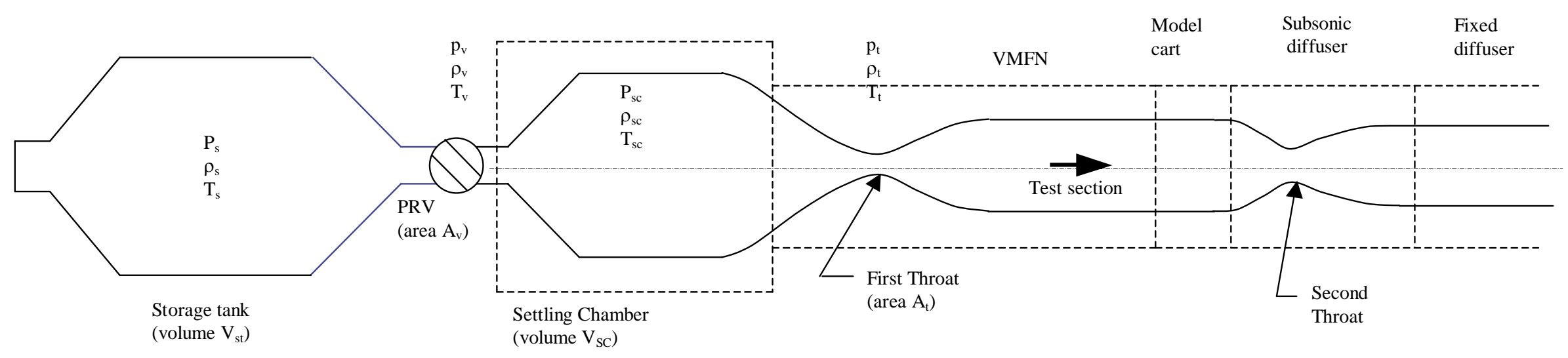

Fig. 1: Schematic of 0.6m wind tunnel with VMFN 


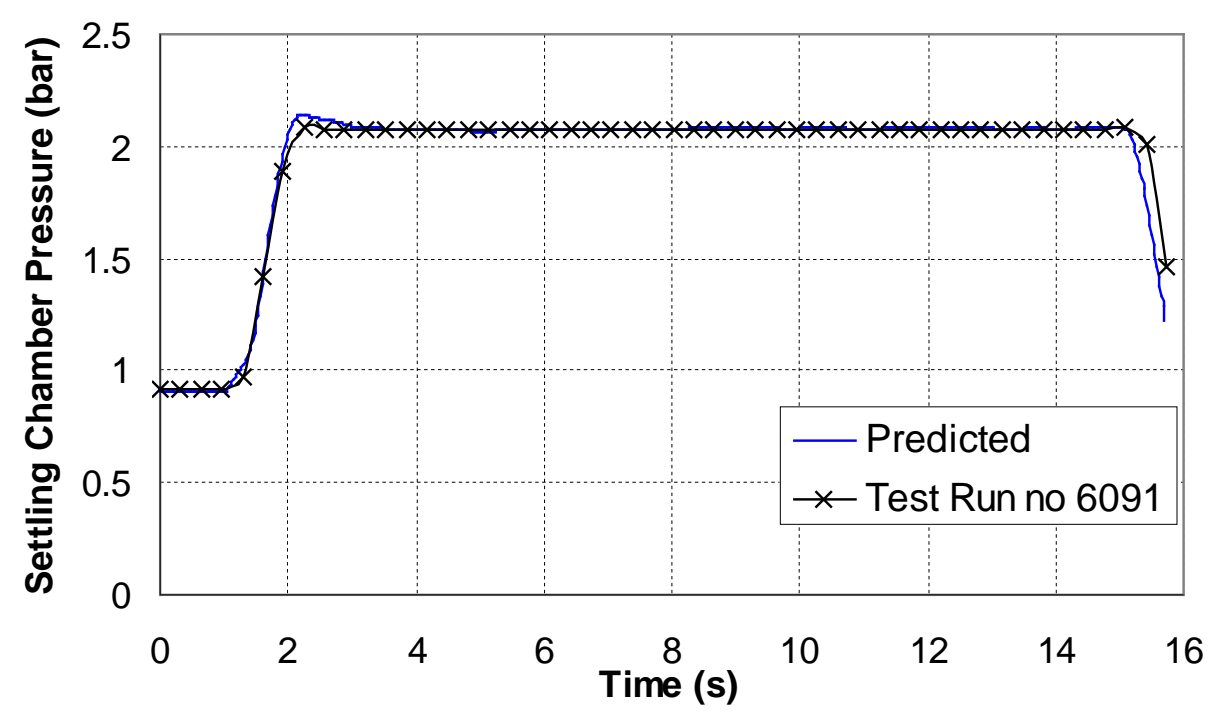

Fig. 2: Comparis on of experimental results of settling chamber pressure characterictics with theoritical prediction for $\mathrm{M}=0.5$

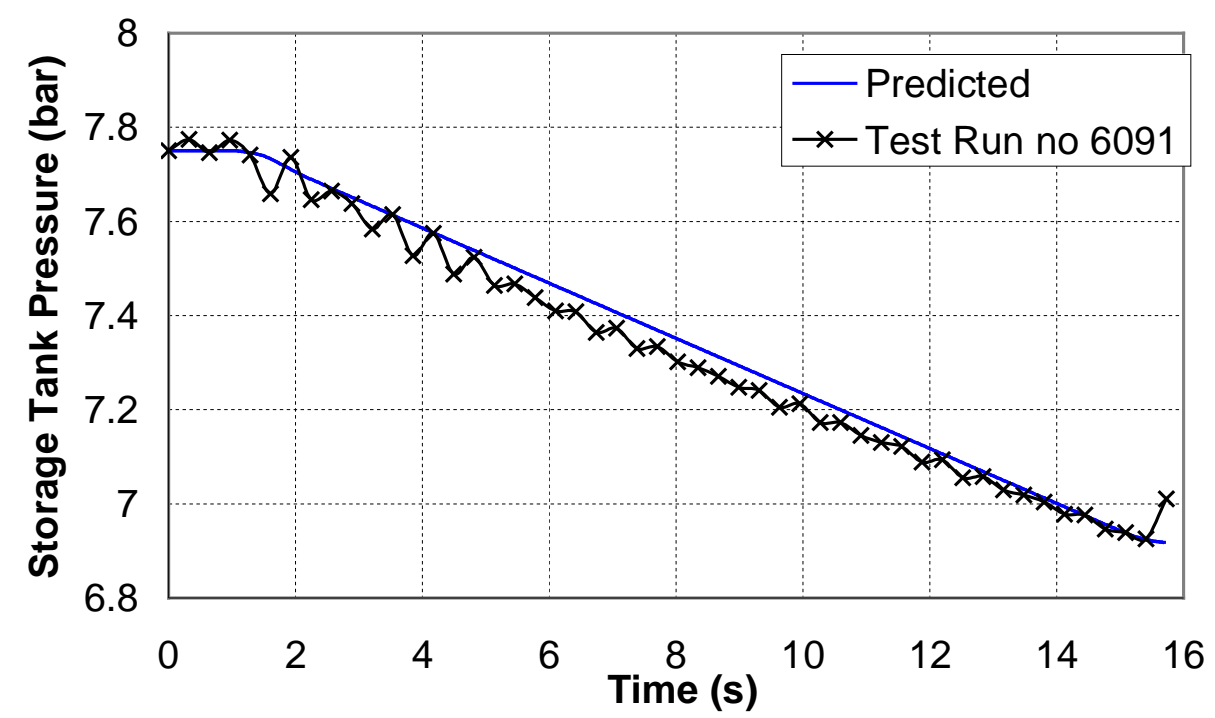

Fig. 3: Comparison of experimental results of storage tank pressure drop with theoritical prediction for $\mathrm{M}=\mathbf{0 . 5}$ 


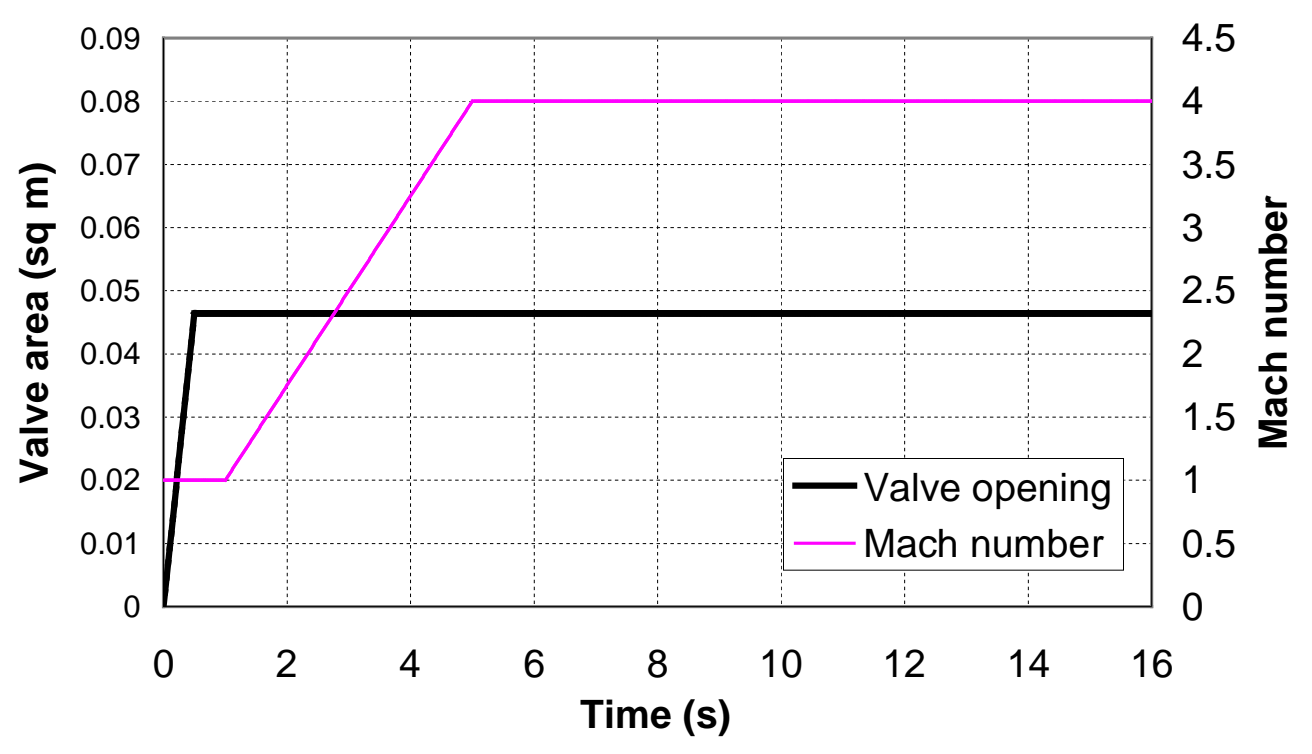

Fig. 4: Inputs of PRV trajectory and Mach number profile

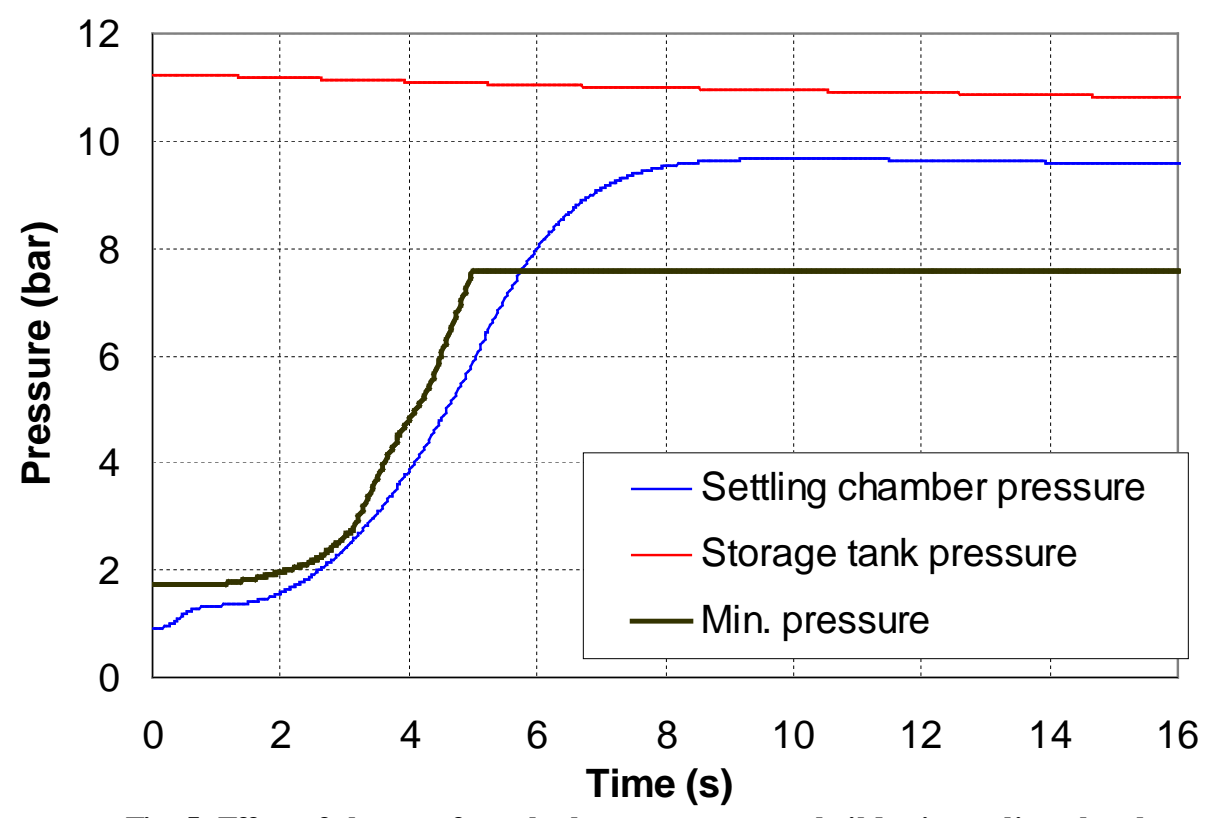

Fig. 5: Effect of change of nozzle throat on pressure buildup in settling chamber 


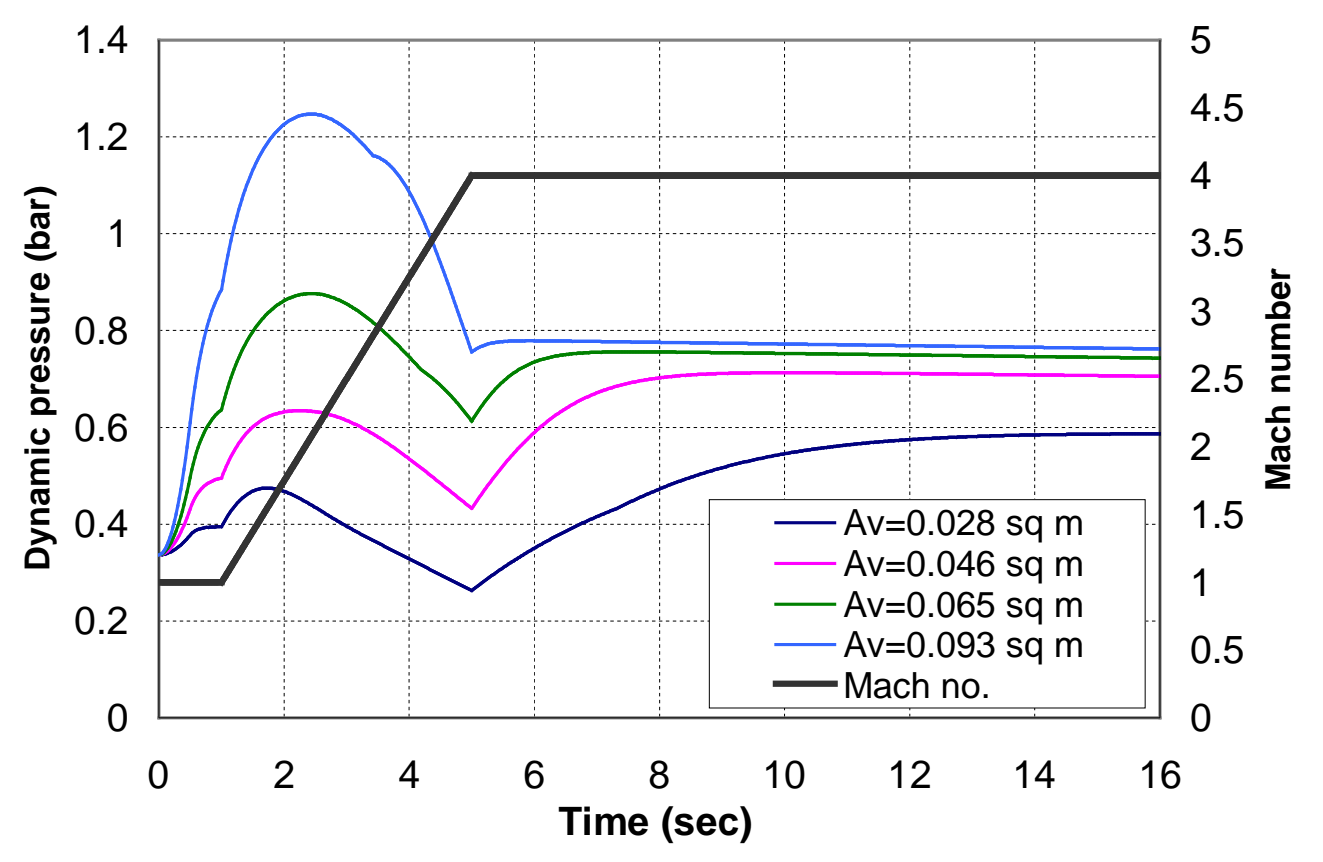

Fig. 6: Effect of change of nozzle contour on dynamic pressure for some valve openings

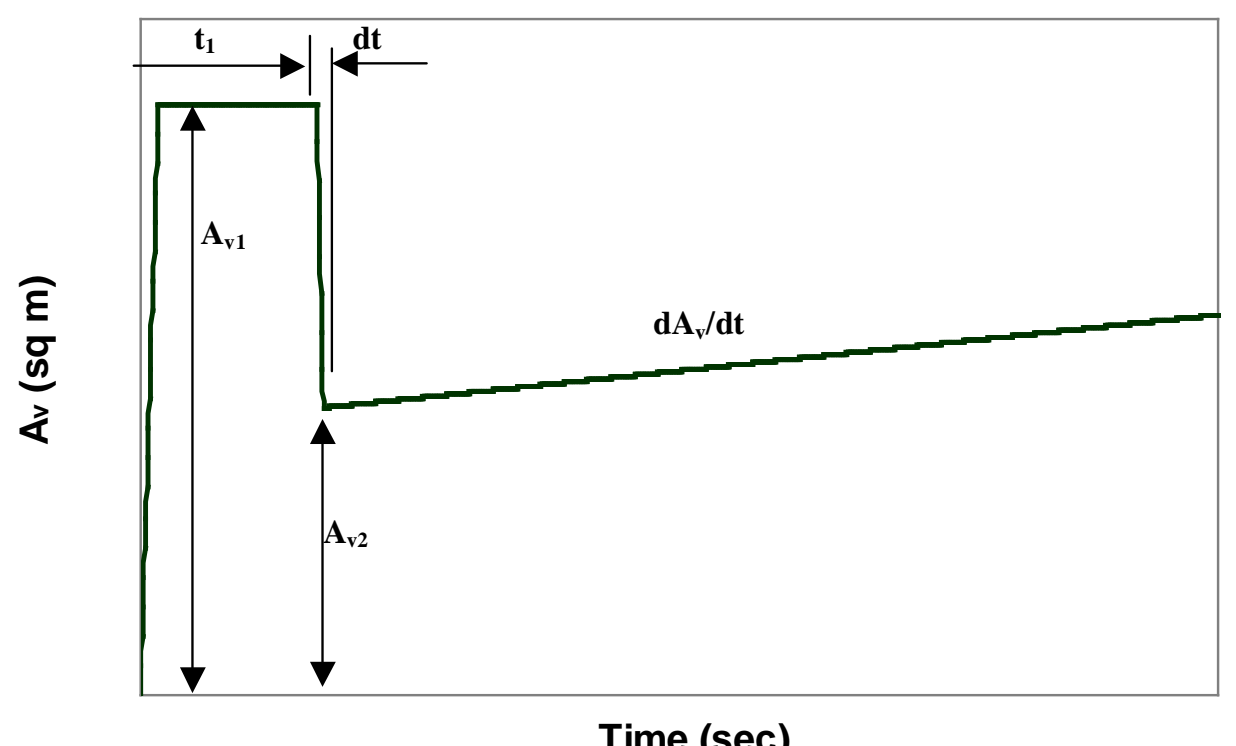

Fig. 7: Typical valve opening trajectory in regulated mode 